\title{
Reopening of elective total joint replacement surgery in a high-volume single-specialty hospital within a COVID-19 epicenter
}

\author{
Taryn E. LeRoy ${ }^{1,2}$, Raminta V. Theriault ${ }^{1,2}$, Nathan J. Sinz ${ }^{1,2}$, Gabriel S. Perrone ${ }^{1,2}$, Christopher J. Fang ${ }^{2}$, \\ David A. Mattingly ${ }^{2}$, Eric L. Smith ${ }^{2}$ \\ ${ }^{1}$ Department of Orthopaedic Surgery, Tufts Medical Center, Boston, MA, USA; ${ }^{2}$ Department of Orthopaedic Surgery, New England Baptist \\ Hospital, Boston, MA, USA \\ Contributions: (I) Conception and design: All authors; (II) Administrative support: TE LeRoy, RV Theriault, GS Perrone, CJ Fang, DA Mattingly, EL \\ Smith; (III) Provision of study materials or patients: TE LeRoy, CJ Fang, DA Mattingly, EL Smith; (IV) Collection and assembly of data: TE LeRoy, \\ RV Theriault, NJ Sinz, GS Perrone, CJ Fang; (V) Data analysis and interpretation: TE LeRoy, RV Theriault, NJ Sinz, GS Perrone; (VI) Manuscript \\ writing: All authors; (VII) Final approval of manuscript: All authors. \\ Correspondence to: Eric L. Smith. 125 Parker Hill Ave, Boston, MA 02120, USA. Email: esmith@nebh.org.
}

Background: The coronavirus disease (COVID-19) pandemic has created significant change globally in healthcare systems. With the recommendation to stop elective surgery, healthcare systems incurred significant financial losses. As the initial surge begins to decline, hospitals and surgery centers are devising plans to resume elective cases. Therefore, the aim of this study is to describe an approach to resuming elective surgery for total joint replacement at our single-specialty, orthopaedic-only institution.

Methods: With our multidisciplinary team, our hospital created an approach to resume elective surgery for total joint replacement under the COVID-19 pandemic conditions and state regulations. We describe our steps and processes to remain a COVID-19 negative institution.

Results: Through our approach, our orthopaedic-only specialty institution was able to return to elective total joint arthroplasty procedures at significant volume during the pandemic, and, to date, has remained COVID-19 negative through these efforts.

Conclusions: This manuscript aims to summarize an approach to the resumption of elective surgery focusing on four main areas of care: (I) preoperative screening and care, (II) operative care, (III) immediate post-operative care, and (IV) inpatient concerns. Our example may serve as a basic model which can be used as a template and customized to meet the specific needs and restrictions of other institutions as they work through returning to baseline.

Keywords: Coronavirus disease (COVID-19); SARS-CoV-2; pandemic; arthroplasty

Received: 24 November 2020; Accepted: 28 February 2021; Published: 25 June 2021.

doi: 10.21037/jhmhp-20-149

View this article at: http://dx.doi.org/10.21037/jhmhp-20-149

\section{Introduction}

The coronavirus disease (COVID-19) caused by the SARS$\mathrm{CoV}-2$ virus has created global healthcare and economic crises. In Massachusetts, healthcare facilities were advised to postpone elective surgeries on March 15, 2020 with the goal of preserving critical supplies, including personal protective equipment (PPE), ventilators, and optimizing the number of beds available (1). While undoubtedly necessary, the postponement of high revenue generating elective procedures placed significant financial strain on healthcare systems. This afforded orthopaedic surgeons the opportunity to rethink current healthcare models, restructure practices, and find new methods to enhance patient care. While healthcare systems devise strategies to resume elective surgeries, input from federal and state governments, the Center for Disease Control (CDC), the American Academy of Orthopaedic Surgeons (AAOS) (2), 
the American Association of Hip and Knee Surgeons (AAHKS) (3), and industry partners have provided additional resources and guidance.

Our institution is a high-volume, COVID-19 negative, orthopaedic-only specialty hospital in Boston, Massachusetts that performs approximately 14,000 orthopaedic surgery cases per year. Total joint arthroplasty, including shoulder arthroplasty, constitutes approximately 65 percent of the inpatient case volume. During the majority of the period of cessation of surgical cases between March 17 and May 3, 2020, estimates suggested cancellation of 913 inpatient and 558 outpatient surgical procedures. Our institution estimates a monthly $\$ 9,000,000$ loss in revenue. Due to a significant decrease in volume, our institution offered to house various non-COVID-19 patients from other institutions to offload neighboring hospitals involved in the care of COVID-19 patients, thus remaining a COVID-19 negative hospital during the pandemic. The COVID-19 negative status of the hospital was also maintained due to the lack of an emergency department at our institution.

As the number of hospitalized COVID-19 patients continues to decline, our institution created guidelines for resuming elective surgery (4). Utilizing the aforementioned resources, this guideline represents a culmination of best practices designed by the hospital's multidisciplinary committee. The goal of this protocol is mitigating risk to patients and staff while optimizing efficiency when resuming elective surgery. The purpose of this manuscript is to document the best practices and guidelines for restarting elective surgery at a high-volume, COVID-19 negative, orthopaedic-only specialty hospital as a model for other institutions.

\section{Methods}

\section{General considerations}

There are several important considerations when designing a protocol for the reimplementation of elective surgery during the COVID-19 pandemic. First, collaboration is necessary among several specialties and hospital departments. The surgeon-in-chief and chief nursing and operating officer formed a steering committee overseeing specific work groups consisting of physicians from medicine, infectious disease, and orthopaedics as well as operating room and inpatient nursing, pharmacy, and administrative personnel including the hospital president and CEO. Subcommittees specifically focused on 4 different areas: (I) preoperative screening and care, (II) operative care, (III) immediate post-operative care, and (IV) inpatient concerns. Through this collaboration, a comprehensive protocol for the staged reintroduction of elective surgery was developed.

Following creation of a protocol, next steps involve implementing such changes in an efficient and safe manner. The protocol will be distributed to all hospital employees and made available electronically for review. There will be significant logistical changes in the workflow and floor traffic in all areas of the hospital, including outpatient preoperative evaluation, travel to and within the hospital, operating room workflow, and post-operative care. In order to implement these changes, several considerations with regards to staffing will be made. There will be increased use of telemedicine encounters and use of iPads in the hospital to provide patient education while limiting personto-person interactions. Additionally, increased access to disinfectant wipes will be made available, the hospital will be terminally cleaned before resumption of surgery, and virtual education modules will be completed by staff to ensure that all employees are properly trained on new protocols. All staff members will be electronically screened daily via text message and will only be allowed to work if asymptomatic (Figure 1). Symptoms include fever, body aches, cough, sore throat, shortness of breath, vomiting, diarrhea, and loss of smell or taste. In order to encourage workers to stay home if symptomatic, the institution increased employee paid sick leave to eliminate the financial incentive to keep working.

While this protocol's goal is to produce a safe and comprehensive means to reintroduce elective surgery, this protocol is based on the information currently available that will likely continue to evolve. As such, considerable understanding and flexibility will be necessary of all employees, patients, and their caregivers. Weekly meetings among committee members will allow for changes and continual reevaluation of the protocol. The purpose of this initial protocol is to serve as the model for the reintroduction of elective surgery at our institution with the understanding that there will likely be several iterations before the final equilibrium is reached.

\section{Exclusion criteria}

Our institution has a long-standing history of preoperative screening for elective surgery. Beginning in 1997, through collaboration with medicine subspecialists, each patient is systematically evaluated before surgery. Prior to the COVID-19 pandemic, this included an inperson evaluation and risk stratification based on medical 


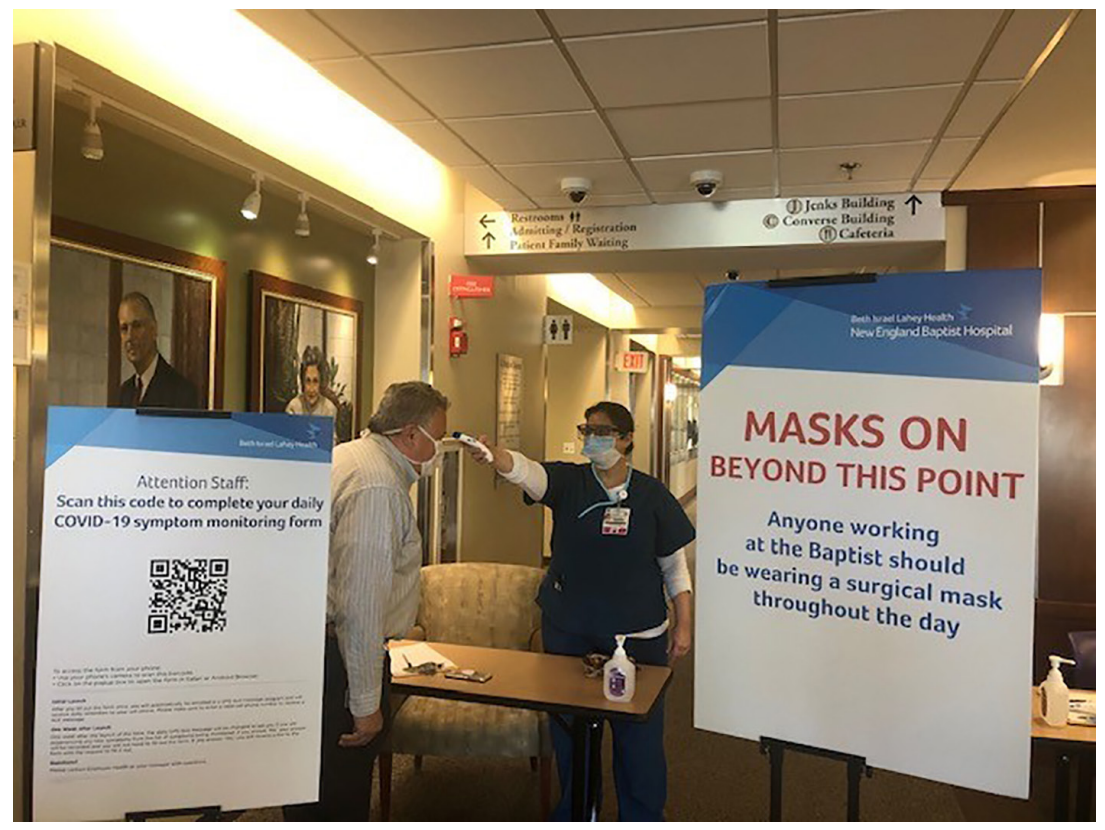

Figure 1 Staff entrance checkpoint with symptom monitoring and temperature check.

comorbidities. Further evaluation and testing were then done as needed with evaluation and clearance obtained from the necessary subspecialties. The results were then documented and reviewed through the prescreening department weekly. COVID-19 has increased the need for prescreening of patients to perform elective surgeries, but with the restriction of limiting in-person interactions and unnecessary evaluations. Robust pre-screening processes will continue, but with an increased emphasis on telemedicine evaluations, when appropriate, and the addition of stricter exclusion criteria. For example, the fatality rates for patients with COVID-19 are higher for those with the following comorbidities: 10.5 percent for cardiovascular disease, 7.3 percent for diabetes, approximately six percent each for chronic respiratory disease, hypertension, and cancer (5). The basis for these criteria is derived from the medical literature, recommendation of medical specialists, and recently published studies in the literature (Table 1) (6-12). As more information becomes available about specific patient risk factors for poor outcomes with COVID-19 infection, these exclusion criteria will likely change $(13,14)$.

\section{Results}

\section{Staging return phases and testing}

In order to proceed with elective surgery, risk to staff and patients must be mitigated by appropriately using PPE. Four phases to restart elective surgery were developed, which allows changes to be enacted to the ongoing COVID-19 pandemic and implement appropriate PPE, testing, and screening protocols (Table 2). These phases are decided on by the governor of Massachusetts and his Reopening Advisory Board. In addition, the president of the hospital held daily meetings with the state department of public health to ensure up-to-date information was utilized. Phase 1 resembles circumstances during the surge of COVID-19, with limited testing availability and ongoing community transmission. Phase 2 represents reduced community transmission with improved testing availability. Both PCR testing and antibody testing for asymptomatic individuals are widely available. Antibody testing studies will help provide a better understanding of disease transmission. Phase 3 is characterized by predictable transmission within the community with disease transmission mostly limited to high risk groups. In phase 3 , rapid point of care testing is available. Phase 4 represents a time similar to the preCOVID-19 era where there is widespread availability of a vaccine or herd immunity. In phase 4 , testing returns to as needed and standard precautions are resumed. It is unclear how long each phase will last, but with each phase comes different recommendations for testing and PPE. At this time, all patients are being tested prior to 
Table 1 Exclusion criteria for elective surgery

\begin{tabular}{|c|c|c|c|c|}
\hline Demographics & Cardiac & Pulmonary & Other comorbidities & Patient factors \\
\hline ASA IV & $\begin{array}{l}\text { Coronary artery stent: bare } \\
\text { metal within } 45 \text { days; DES } \\
\text { within } 6 \text { months }\end{array}$ & $\begin{array}{l}\text { Moderate to severe } \\
\text { COPD }\end{array}$ & Cerebrovascular disease & $\begin{array}{l}\text { A patient requiring rehab } \\
\text { after surgery }\end{array}$ \\
\hline \multirow{2}{*}{$\mathrm{BMI}>40$} & $\begin{array}{l}\text { Hypertrophic cardiomyopathy: } \\
\text { moderate or severe }\end{array}$ & Untreated OSA & Steroid therapy & Cognitive deficiency \\
\hline & $\begin{array}{l}\text { Moderate or severe valvular } \\
\text { disease }\end{array}$ & $\begin{array}{l}\text { Pulmonary hypertension } \\
(\text { PAP }>45)\end{array}$ & $\begin{array}{l}\text { Immunosuppression } \\
\text { therapy }\end{array}$ & \\
\hline
\end{tabular}

surgery; however, phases may vary at other institutions based on testing availability in their community. Under the protocol, if a patient is COVID-19 positive by PCR, regardless of antibody testing, the recommendation is to postpone elective surgery. Although there is an absence of high-level evidence reporting complications and worseoutcomes in COVID-19 positive patients, it is currently the general consensus and ethical approach to delay surgery until a patient is disease free. Special considerations would be made for urgent/life-threatening cases in the setting of COVID-19 positive PCR.

\section{Prior to day of surgery (pre-admission screening, pre-op considerations)}

The surgeon office will contact patients directly to identify eligible patients willing to undergo surgery. Patients will be informed that post-operative rehabilitation and home health services may be limited or unavailable and patients are advised to make necessary plans for additional family support. If the patient is deemed eligible and willing to proceed with surgery, the Surgeon Office contacts the Pre-Admission Screening Unit (PASU) for appointment scheduling. The patient will complete an electronic intake form via a secure web platform which will be subsequently reviewed by the PASU (Table 1). Patients that meet exclusion criteria will be identified in PASU and subjected to an interdisciplinary (i.e., anesthesia, nursing, hospitalist, orthopaedic surgery) case review to determine if surgery can proceed. Patients will undergo COVID-19 testing 48 hours before surgery according to the phased approach as discussed above based on community transmission and testing availability (Table 2). After COVID-19 testing, all patients will be instructed to self-quarantine to avoid additional exposure. Patients that have completed the PASU intake form in advance and determined to be low risk may be eligible for a telemedicine interview and subsequent radiographs and labs. The history and physical will be completed on the day of surgery. If a patient does not pass their telemedicine interview, or they are not deemed low risk, they will be required to attend an in-person appointment with PASU.

Prior to arriving to the hospital, patients will be preregistered by the Patient Access department by phone to review the current safety protocol, social distancing guidelines, and to obtain verbal consents (Figure 2). Patients will be instructed to travel to the hospital for the PASU appointment alone or with one support person, arriving 1530 minutes before the appointment. Patients will call the Patient Access team in the PASU upon arrival. They will remain in their car until they receive instruction to proceed to the lobby with assistance of a transport team as needed. If the patient requires imaging, this will be performed prior to visiting the PASU. There is one main entrance for all patients and a separate entrance for hospital staff (Figure 1). Entrances and high contact areas are cleaned using a 


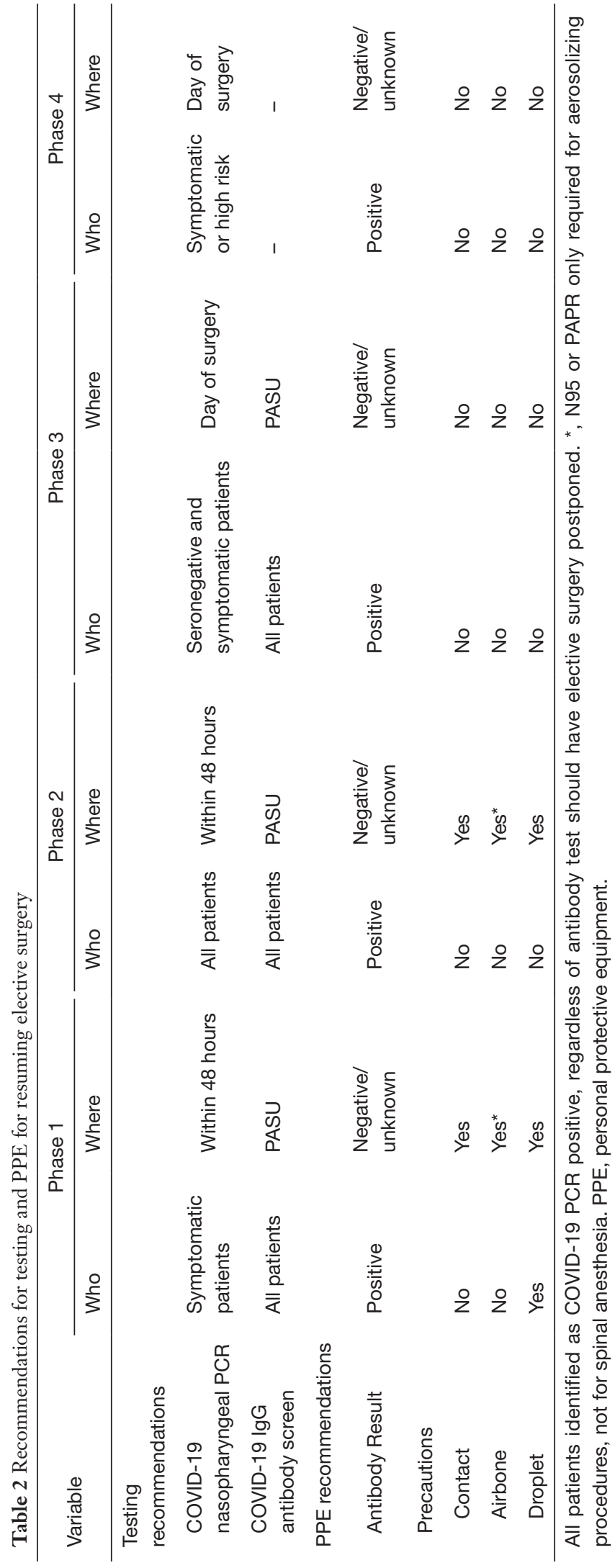

standard protocol with increased frequency. All lobbies and meeting spaces will be reconfigured for social distancing. Ideally, no patients will wait in the Patient Access lobby prior to proceeding to the PASU for screening. Additional socially distanced waiting areas will be provided for patients requiring radiology and preoperative lab work. Once any necessary imaging is complete, the Patient Access department will register the patient in the examination room for the PASU appointment. Consults with other relevant departments will be available for both in office and telehealth visits when appropriate.

Surgeons will be provided a stratified list of patients seen in the PASU with their screening status: green, yellow, or red. Green refers to patients cleared with no exclusion criteria who may proceed with scheduled surgery. Yellow refers to patients requiring additional follow-up and/or inter-disciplinary review with PASU clearance pending. Red refers to patients that were not cleared by PASU to proceed with surgery. Each surgeon will then submit a preferred surgical order one week prior to surgery for review. Ideally, patients with a tentative same day home discharge plan will be prioritized earlier in the day with inpatient cases to follow. In preparation for the day of surgery, the Patient Access department contacts each patient the day before surgery to inquire about any specific COVID-19 symptoms utilizing a pre-screening template. If the patient reports any symptoms, the patient is subsequently contacted within the hour by a nurse for further screening and determination if any further testing is required before surgery. Upon arrival on the day of surgery, all patients will follow the same protocol as noted above. After registration is complete, the pre-operative assessment by the nurse, anesthesia team, and orthopaedic team will be performed in order to proceed with surgery.

\section{Day of surgery (operating room and $P A C U$, inpatient and discharge)}

Our hospital traditionally has 16 operating rooms functioning daily on weekdays and has the capacity to open rooms on the weekend for urgent and emergent cases. Due to the additional staffing demands and logistical restraints of the added safety precautions, we will start with opening 12 operating rooms daily. To account for the relative decrease in weekday operating room availability, there will be an option for opening more operating rooms on Saturday by surgeon preference and availability. Operating room start times will be staggered such that four rooms each will begin at 7:00 $\mathrm{AM}, 7: 30 \mathrm{AM}$, and 8:00 $\mathrm{AM}$. No elective surgeries 
1. New England Baptist Hospital COVID-19 Questionnaire/Algorithm (Use for front desk/check-in/telephone screenings, return to healthcare setting for patients and patients/visitors/escorts with prior COVID-19)

Beth Israel Lahey Health

New England Baptist Hospital
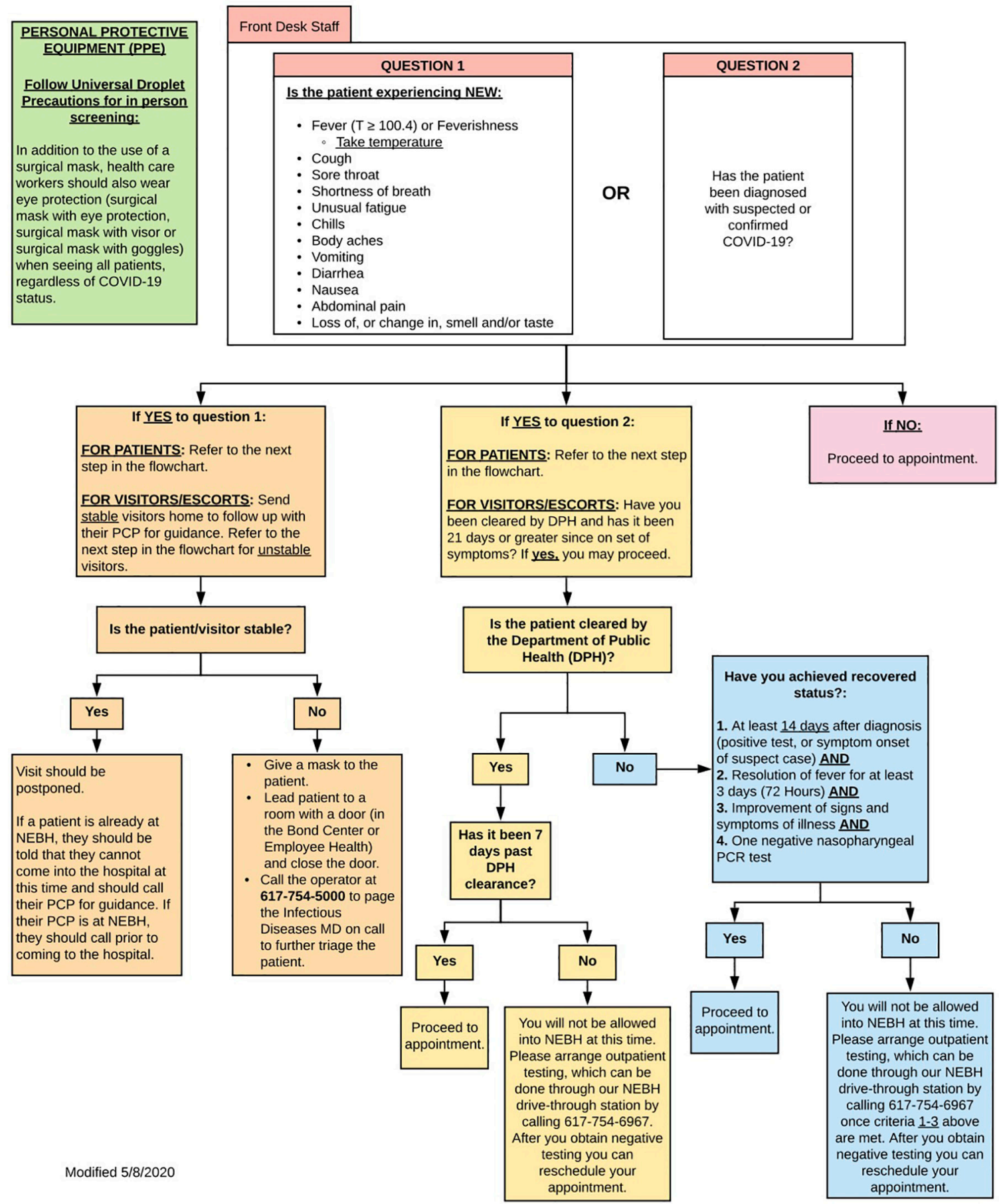

Figure 2 Questionnaire algorithm. 
will be scheduled to begin after 3:30 PM. There will be one runner assigned for every three rooms to limit OR traffic. If PPE contact precautions are able to be lifted, sixteen rooms will run with six rooms beginning at 7:00 AM, six rooms at 7:30 AM and four rooms at 8:00 AM. The number of people will be limited in each OR to reduce the frequency of door openings. Room equipment will be kept to a minimum. Cases involving higher risk patients with airway concerns based on preoperative risk stratification will be performed in the morning. To optimize efficiency during the reopening phase, there will be strict guidelines regarding the timeliness of adhering to scheduled surgical start times as swing rooms may not be available during the early stages of reopening. If the surgeon is more than 15 minutes late to the OR and they are the primary reason for the delay in surgery, they will be given a warning for their first offense and will lose two weeks of block time for a second offense. Spinal anesthesia will be the preferred form of anesthesia when appropriate and these cases will be performed earlier in the day to maximize postoperative time for spinal anesthesia effects to diminish and increase early mobility for any patients suitable to be discharged on the same day as surgery. For patients requiring general anesthesia, rapid sequence intubation with succinylcholine per American Society for Anesthesiologists guidelines during the COVID-19 pandemic will be performed (15). All patients will be treated with contact precautions and droplet precautions when appropriate (Table 2). All patients receive a standardized periarticular injection of 0.5 per bupivacaine $(50 \mathrm{~mL})$ mixed with $30 \mathrm{~mL}$ of sterile saline and $30 \mathrm{mg}$ of ketorolac (unless contraindicated). Operating room turnover will begin 30 minutes after extubation to further protect employees. Vendor access will be limited in the OR and cases will be limited to one vendor when possible. Patients appropriate for outpatient surgery will recover in the day surgery recovery area. When an eligible outpatient surgery patient is deemed ready for discharge, a designated escort will bring them to their family member at the front of the hospital to minimize family members entering the hospital. Patients will be spaced accordingly in PACU to maintain social distancing recommendations. Any patient that remains intubated will recover in a designated isolation room. Patients being admitted will be transferred to the floor when clinically appropriate to private rooms. Patients meeting criteria for "short stay" will be located on 2 specific floors to maximize efficiency. Staffing hours will be adjusted as needed to accommodate for patients requiring longer stays due to lack of rehabilitation center bed availability.
All patients receive a standardized post-operative pain protocol. Furthermore, prior to surgery, post-operative DVT prophylaxis is selected by the surgeon based on preoperative risk assessment and is filed in the patient's chart for ease of post-operative order sets. All post-operative order sets and guidelines for discharge will be standardized. Discharge instructions will be available on iPad for patient review to limit face-to-face interactions with staff members.

\section{Monitoring success}

Once resuming elective surgery, we plan to monitor our progress, success, and failures through a multi-faceted approach. We will compare pre- and post-COVID-19 patients with regard to baseline patient demographics, length of hospital stay, complications, and outcome measures. We will also evaluate financial recovery once restarting elective surgery. We anticipate a decrease in ability for patients to be discharged to rehabilitation facilities as well as obtain in-home rehab services. This may lead to decreased patient satisfaction and increased complication rates due to lack of professional support once discharged from the hospital. Primary outcome measures will include 90-day complications, readmissions and need for further surgery. Secondary outcome measures will include patient satisfaction regarding their in-hospital experience and also post-operative care once discharged, as well as number of patients testing positive for COVID-19 within two weeks of surgery and the proportion of staff who tested positive. These will be assessed by way of two distinct electronic surveys. It is expected that there may be a variety of differences in the pre- and post-COVID-19 patients. Our goal is to learn from our initial experience in resuming elective surgery, implement appropriate changes in this dynamic process and continue to provide excellent orthopaedic care to our patient population.

\section{Conclusions}

\section{Final thoughts}

The COVID-19 pandemic has created widespread changes throughout the healthcare system. It has allowed orthopaedic surgeons the opportunity to rethink current healthcare models, restructure practices, and enhance care for patients. As a high-volume orthopaedic specialty hospital, the restrictions on elective surgeries during the pandemic have resulted in a significant number of cases 
being cancelled and postponed. The ability to resume elective surgery in a safe environment is critical to ongoing care of patients. The economic implications of resuming surgery at our institution is also significant given the high volume performed and reliance on surgical procedures as a primary income source. The creation of a guideline for the resumption of elective surgery is the culmination of best practices. Through the input of federal, state, and local governments, AAOS, AAHKS, industry, and a multidisciplinary clinical committee, this protocol is a comprehensive approach to mitigating risk to patients and staff while optimizing efficiency when resuming elective surgery. While each healthcare system creates their own protocols, this proposal can provide other groups a resource from which to formulate their guidelines. Our institution is in a unique situation where it has remained a COVID-19 negative hospital. As an orthopaedic specialty hospital without an emergency room, the scope of care of our institution is outside of the scope required to treat patients with COVID-19. Within our larger hospital network, our institution was tasked with accepting transfers of patients who were COVID-19 negative in order to free up hospital beds for our partners treating COVID-19. The term we use is only meant to describe the infrastructure in our hospital as well as lack of patients presenting to us in a non-elective fashion, which provides the opportunity to rapidly increase surgical volume as the state reopens.

The development of this protocol through a multidisciplinary committee has also enabled improvement in several aspects of orthopaedic care. This is especially evident through the standardization of spinal anesthesia, pain management, and anticoagulation protocols for patients undergoing TJA, which will likely improve the care of our patients beyond the pandemic. Furthermore, the continued engagement of the committee will aid in continually reexamining and adapting this protocol throughout the process of reintroducing elective surgical cases. It is impossible to design a flawless protocol with seamless implementation during such a dynamic process as the COVID-19 pandemic. As recommendations regarding care and preventing the spread of disease, this protocol will change and adapt to accommodate the most current guidelines. The communication of these changes will be made to employees and patients virtually to limit large in-person gatherings. As COVID-19 is a global pandemic and strategies exists throughout the world for a return to elective surgery (16), our protocol represents an example of a United States hospital where domestic regulations and rates of COVID-19 differ. Further, our protocol represents a single-specialty orthopaedic-only institution with no emergency roomand our model may apply more or less depending on the nature of other hospitals. But we believe our example may be valuable for others to consider.

There are certain limitations when applying this protocol to other institutions. Our institution is a unique, orthopaedic-only specialty hospital without an emergency department, which has remained COVID-19 negative. Furthermore, our institution has a long-standing history of extensive, collaborative, multi-department pre-screening protocols and medical optimization of patients prior to surgery. This has led to efficiency and overall excellent outcomes for patients undergoing TJA, which may put our institution at an advantage when instituting some of the changes in the protocol. Compared to an academic medical center or community hospital, our institution is more similar to certain high-volume orthopaedic surgical centers and therefore this protocol may be more applicable as a model for such institutions for resuming elective surgery. This proposal may not be relevant in its entirety to every institution; however, it may serve as a basic model which can be customized to meet the specific needs and restrictions of other institutions.

\section{Acknowledgments}

We thank Brian L. Hollenbeck, MD, Jonathan Shaker, MS, Mary Sullivan Smith, DNP, RN, NEA-BC, and Scott G. Tromanhauser, MD for contributing to the project.

Funding: None.

\section{Footnote}

Data Sharing Statement: Available at http://dx.doi. org/10.21037/jhmhp-20-149

Peer Review File: Available at http://dx.doi.org/10.21037/ jhmhp-20-149

Conflicts of Interest: All authors have completed the ICMJE uniform disclosure form (available at http://dx.doi. org/10.21037/jhmhp-20-149). Dr. ELS is a paid consultant and receives research support from Depuy and Conformis. Dr. DAM received royalties from Depuy and is a paid and unpaid consultant for Depuy. The other authors have no conflicts of interest to declare. 
Ethical Statement: The authors are accountable for all aspects of the work in ensuring that questions related to the accuracy or integrity of any part of the work are appropriately investigated and resolved. The study was waived by the Institutional Review Board and written informed consent was obtained from all patients.

Open Access Statement: This is an Open Access article distributed in accordance with the Creative Commons Attribution-NonCommercial-NoDerivs 4.0 International License (CC BY-NC-ND 4.0), which permits the noncommercial replication and distribution of the article with the strict proviso that no changes or edits are made and the original work is properly cited (including links to both the formal publication through the relevant DOI and the license). See: https://creativecommons.org/licenses/by-nc-nd/4.0/.

\section{References}

1. Mass.gov. COVID-19 State of Emergency [Internet]. Available online: https://www.mass.gov/info-details/covid19-state-of-emergency

2. Guy D, Bosco JA, Savoie F. AAOS Guidelines for Elective Surgery During the COVID-19 Pandemic [Internet]. AAOS.org 2020. Available online: https://www.aaos.org/ about/covid-19-information-for-our-members/aaosguidelines-for-elective-surgery/

3. Parvizi J, Gehrke T, Ca K, et al. Erratum: Resuming Elective Orthopaedic Surgery During the COVID-19 Pandemic. J Bone Joint Surg Am 2020;102:e113.

4. Stening T. Rate of positive coronavirus cases in Massachusetts declining in May, Gov. Charlie Baker says as officials announce 132 new death, 1,696 new cases [Internet]. Masslive.com 2020. Available online: https:// www.masslive.com/coronavirus/2020/05/rate-of-positivecoronavirus-cases-in-massachusetts-declining-in-may-govcharlie-baker-says-as-officials-announce-132-new-deaths1696-new-cases.html

5. Yang S, Cao P, Du P, et al. Early estimation of the case fatality rate of COVID-19 in mainland China: a data-

doi: 10.21037/jhmhp-20-149

Cite this article as: LeRoy TE, Theriault RV, Sinz NJ, Perrone GS, Fang CJ, Mattingly DA, Smith EL. Reopening of elective total joint replacement surgery in a high-volume singlespecialty hospital within a COVID-19 epicenter. J Hosp Manag Health Policy 2021;5:13. driven analysis. Ann Transl Med 2020;8:128.

6. Gupta PK, Gupta H, Sundaram A, et al. Development and validation of a risk calculator for prediction of cardiac risk after surgery. Circulation 2011;124:381-7.

7. Boersma E, Kertai MD, Schouten O, et al. Perioperative cardiovascular mortality in noncardiac surgery: validation of the Lee cardiac risk index. Am J Med 2005;118:1134-41.

8. Gov.uk. Guidance on shielding and protecting people who are clinically extremely vulnerable from COVID-19 [Internet]. Available online: https://www.gov.uk/ government/publications/guidance-on-shielding-andprotecting-extremely-vulnerable-persons-from-covid-19/ guidance-on-shielding-and-protecting-extremelyvulnerable-persons-from-covid-19

9. Chen T, Wu D, Chen H, et al. Clinical characteristics of 113 deceased patients with coronavirus disease 2019: Retrospective study. BMJ 2020;368:m1091.

10. Grasselli G, Zangrillo A, Zanella A, et al. Baseline Characteristics and Outcomes of 1591 Patients Infected with SARS-CoV-2 Admitted to ICUs of the Lombardy Region, Italy. JAMA 2020;323:1574-81.

11. Wang $\mathrm{L}, \mathrm{He} \mathrm{W}, \mathrm{Yu} \mathrm{X}$, et al. Coronavirus disease 2019 in elderly patients: Characteristics and prognostic factors based on 4-week follow-up. J Infect 2020;80:639-45.

12. Guan WJ, Liang WH, Zhao Y, et al. Comorbidity and its impact on 1,590 patients with Covid-19 in China: A nationwide analysis. Eur Respir J 2020;55:2000547.

13. Chen N, Zhou M, Dong X, et al. Epidemiological and clinical characteristics of 99 cases of 2019 novel coronavirus pneumonia in Wuhan, China: a descriptive study. Lancet 2020;395:507-13.

14. Jordan RE, Adab P, Cheng KK. Covid-19: risk factors for severe disease and death. BMJ 2020;368:m1198.

15. ASAHQ.org. In the Spotlight: COVID-19 Information [Internet]. Available online: https://www.asahq.org/in-thespotlight/coronavirus-covid-19-information

16. Zorzi C, Piovan G, Screpis D, et al. Elective Orthopaedic Surgery During COVID-19. JBJS Open Access 2020;5:e20.00084. 